\title{
USO DE INTERPOLACIONES EN TIEMPO REAL COMO UNA NUEVA HERRAMIENTA PARA EVALUAR LOS COSTOS ENERGÉTICOS PARA LA PRODUCCIÓN DE PAPA ENDIFERENTES ESPACIOS DE CULTIVO. UNA HERRAMIENTA PARA PRODUCCIÓN LIMPIA
}

\section{Using of interpolations in real time as new tool to evaluate the energy costs in the production of pope in different cultivation spaces. A tool for Clean Production}

\author{
FERNANDO FELIPE MUÑOZ1 \\ 1Universidad del Cauca
}

E-mail: fernandofelipemunoz@gmail.com

\section{Resumen}

Se presenta un análisis que evalúa las necesidades y costos de energía (agroquímicos) para la producción de papa mediante un análisis de distribución en diferentes pendientes de cultivo. Se desarrolla un concepto de interpolación de energía como herramienta para evaluar los nutrientes a través de la pendiente. Las interpolaciones son un método gráfico para integrar de manera precisa múltiples evaluaciones de distancia, de modo que se puede determinar una unidad en una distancia determinada. Finalmente, se evalúan las estrategias para una distribución justa según la pendiente y distancia a los cultivos de papa y para mantener un menor impacto en el agro ecosistema.

Palabras claves: Interpolaciones, Nutrientes, Escorrentía, Micro sensores.

\section{Abstract}

An analysis is presented which evaluate the energy needs and costs (agrochemical) for the production of potato by an analysis of distribution in different cultivation inclinations. A concept of interpolation of energy like a tool to evaluate the nutrients through the slope is developed. The interpolations are a graphic method to integrate in a precise way multiple distance evaluations, so one can determine a unit in a certain distance. Finally, strategies are evaluated for a fair distribution according to the slope and distance to potato cultivations to keep a smallest impact in the agro ecosystem.

Keywords: Interpolation, Nutrients, Runoff, Micro sensors. 


\section{INTRODUCCIÓN}

La agricultura de precisión es el sistema de producción que promueve prácticas de gestión de variables dentro de un campo de acuerdo a las condiciones del lugar. El concepto se basa en nuevas herramientas y fuentes de información proporcionadas por las tecnologías modernas, tales como dispositivos de monitorización del rendimiento, del suelo y de la planta, sensores de plagas y aparatos de teledetección. A pesar de los beneficios, existen restricciones en la tasa de adopción de esa diversidad de herramientas tecnológicas, que varían considerablemente de país en país y de región en región (Seelan et al. 2003).

Las herramientas de precisión espacial brindan soporte para la toma de decisiones en el manejo de sistemas agroforestales. Estas herramientas de decisión permiten abordar la variabilidad espacial y temporal en la demanda de los cultivos y el suministro de nutrientes con una dispersión automatizada y precisa para el control y uso eficiente de insumos (por ejemplo, Kim et al. 2008, Peters \& Evett 2008). Simultáneamente con la evolución tecnológica, la precisión en el uso de agroquímicos aumenta, por cuanto se ha producido una creciente conciencia de que en muchas situaciones lo mejor es variar la cantidad de agroquímicos según la distribución espacial, debido a: (i) las demandas variables de los cultivos, (ii) la variabilidad del tipo de suelo y (iii) las zonas de pendientes. Varios sistemas con un prototipo de aplicación del riego de tasa variable se han desarrollado, pero no han sido sistemas adecuados de apoyo a las decisiones (Sadler et al. 2005). Con el fin de aumentar la funcionalidad en la práctica del riego de precisión, del control en tiempo real, de decisión y de sistemas de control se plantea el siguiente desarrollo teórico.

Esta propuesta incluye un enfoque de modelado (regional o de sitios específicos). La medición directa en los cultivos debe tener en cuenta variables como: estrés hídrico (Green et al. 2006, Peters \& Evett 2008); humedad del suelo (Blonquist et al. 2006, Kim et al. 2008, Vellidis et al. 2008); uso de datos climáticos en tiempo real y un balance de nutriente - suelo para predecir la disponibilidad de nutrientes para el cultivo.

Este modelado tendrá la capacidad de predicción del rendimiento, y la capacidad de programar el riego en tiempo real, donde los datos de calidad estáticos y dinámicos que incluyen los efectos de las lluvias en el sitio específico, zonas de profundidad de enraizamiento, compactación y pendiente son esenciales. Los recientes avances han automatizado el monitoreo de las características agroclimáticas de puntos específicos de monitoreo a herramientas de software de decisión (SIG), vinculados a los sistemas de riego.

Esta investigación actual pretende producir mapas de suelos con variabilidad espacial y temporal diarios del estado del agua nutriente para una gestión simplificada de sistemas de riego y una mayor eficiencia del uso de agua y nutriente (Figura 1).

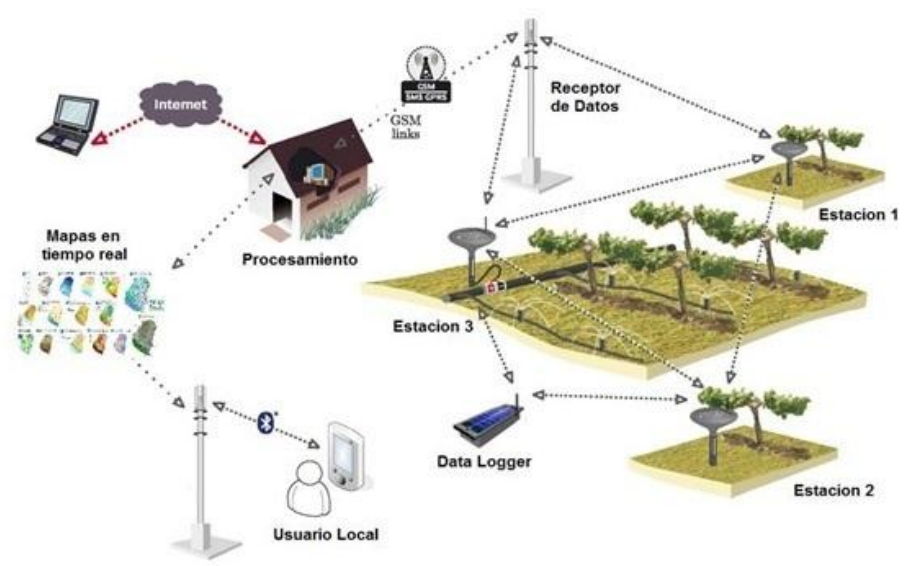

Figura 1. Esquema tecnológico para la adquisición de datos en tiempo real.

En el curso de la evolución tecnológica la miniaturización de dispositivos electrónicos y la tecnología de comunicación inalámbrica han llevado a la aparición de redes de sensores inalámbricos. La potencia necesaria para ejecutar con eficacia los circuitos asociados están siendo reducidos, con la ayuda de la energía obtenida del entorno donde se implementan los dispositivos. En sentido estricto, son arreglos de dispositivos electrónicos con capacidades de detección que están interconectados mediante una red de radio. Existen muchas arquitecturas, que van desde micro-dispositivos integrados con sensores inteligentes para completar la adquisición de dispositivos autoalimentados que el apoyo extienda variedad de sensores externos (Morais et al. 1996). En cuanto al apoyo de la red, muchos protocolos tales como Bluetooth, GSM y formas de propiedad de las interconexiones de redes de radio existentes (Lee et al. 2002).

Hay varias cuestiones clave para hacer frente a la hora de seleccionar la tecnología adecuada para la transferencia inalámbrica de datos. Uno de los criterios más importantes es el soporte de la red, por lo general determinada por el destino de la aplicación, lo que limita la oferta disponible. Otros factores clave son las tasas de transferencia de datos y el consumo de energía. Muchos transceptores de radiofrecuencia independiente son adecuados para el reemplazo del cable (conexiones punto a punto), a menudo usando protocolos propios para mejorar la fiabilidad de transferencia de datos.

\section{METODOLOGÍA}

La disposición de los cultivos de papa está fuertemente condicionada por la pendiente y condiciones ambientales. Además, las características únicas de estos sectores, así como los aspectos topográficos, erodabilidad, dirección de siembra, la disponibilidad de agua y la variación climática a través del día y año, demanda de las herramientas tecnológicas más recientes, tales como el control distribuido y procesamiento de la información. Esto permite ayudar a comprender la variabilidad del cultivo y por lo tanto 
cómo puede ser manejado, lo que mejora la cantidad y calidad del producto. Un buen ejemplo del uso de WSN como una herramienta clave para entender la variabilidad de la productividad se puede encontrar en Camilli et al. (2007). Como un número cada vez mayor de dispositivos electrónicos con varios tipos de sensores están integrados en los procesos agrícolas, integración de sistemas eficientes se ha convertido en un objetivo crítico. La enorme cantidad de datos en campo se pueden adquirir y centralizar en la web, utilizando los sistemas de información geográfica (SIG). A nivel regional, o incluso a escala mundial, la plantación de grandes infraestructuras es el resultado de la interconexión de varias plantaciones locales. Estas plataformas de distribución, que incorpora información de sensores remotos, de las condiciones meteorológicas in situ, niveles de agua, historia del suelo, y de conocimiento de los agricultores acerca de la productividad relativa de algunas "Zonas de Gestión", se puede aplicar, por ejemplo, para predecir el rendimiento y las enfermedades, y para difundir consejos a lo largo de la temporada de cultivo sobre el uso óptimo de agua y los tratamientos químicos necesarios.

Para abordar las cuestiones pertinentes en relación con el despliegue de red, se propone una arquitectura de detección remota de la red, se muestra de forma simplificada en la figura. 1. El diseño de adquisición debe ser auto-sostenible y tiene como objetivo mejorar en el campo de la recopilación de datos y dar soporte de red a los sistemas. Para cada punto de muestreo (estación), se implementa una red de dispositivos para adquirir datos sobre el contenido de humedad del suelo, la temperatura del suelo, temperatura del aire, humedad relativa y radiación solar, entre otros parámetros. Esto crea un nivel intermedio de los nodos de agregación que gestiona las redes de sensores y lleva a cabo la integración de datos locales y las funciones de supervisión, mientras que el mantenimiento de la conectividad a través de toda la región. Dada la ubicación de los cultivos, es necesario contar con tecnologías que permitan la trasmisión de datos aun procesador central. Actualmente se cuentan con tecnologías GPRS y GSM de gran estabilidad que permiten un tráfico constante de información en tiempo y espacio.

La aplicación de tecnología puede proporcionar considerables beneficios financieros para el sector agrícola. Sin embargo, su eficacia depende de la exactitud de la interpolación espacial para definir la variabilidad espacial de las propiedades del suelo. La exactitud de los métodos de interpolación espacial para la predicción de las propiedades del suelo se ha analizado en varios estudios. Kravchenko \& Bullock (1999) con comparaciones del inverso de la distancia (IDW), Kriging ordinario y kriging ordinario log normal de las propiedades del suelo (fósforo (P) y potasio $(\mathrm{K})$ ) de 30 campos experimentales. Encontraron que si el conjunto de datos en virtud del falso es log normal distribuidos y contiene menos de 200 puntos, kriging ordinario log normal generalmente fuera realiza tanto kriging ordinario y IDW, de lo contrario, kriging ordinario es más exitoso.
Además, Laslett et al. (1987) también encontraron Kriging ordinario (isotrópico) como un método mejor que IDW para la interpolación de pH. De hecho, Laslett et al. (1987) consideró que las isolineas con mejor acercamiento a la realidad eran IDW y Kriging. Por el contrario, Gotway et al. (1996) observaron mejores resultados con Kriging para la materia orgánica del suelo y el nitrógeno cuando se utiliza IDW. Weber \& Englund (1992) también encontraron que IDW produce mejores resultados que kriging (con kriging log normal superando kriging ordinario).

\section{CONCLUSIONES}

Este tipo de estudios pueden demostrar que los métodos de predicción espacial utilizada, puede producir resultados en la continua generación de mapas de suelos todo el tiempo y espacio para cada una de las variables. Teniendo en cuenta que según la variable se utilizan diferentes interpoladores para obtener información real desde los datos provenientes de los sensores inalámbricos.

Aunque las técnicas de interpolación no son herramientas de confirmación, si son válidas como unas herramientas de exploración que ayudan en la elección de procedimientos adecuados de interpolación y sus parámetros asociados.

\section{REFERENCIAS}

BLONQUIST, J. M. JONES, S.B. \& ROBINSON, D. A. 2006. Precise irrigation scheduling for turfgrass using a subsurface electromagnetic soil moisture sensor. Agrie. Water Manage 84: 153-165.

CAMILLI, R. D. SAKELLARIOU, B. FOLEY, C. ANAGNOSTOU, A. MALIOS, B. BINGHAM, R. EUSTICE, J. GOUDREAU, K. \& KATSAROS. 2007. Investigation of hydrothermal vents in the Aegean Sea using an integrated mass spectrometer and acoustic navigation system onboard a human occupied submersible. In: Proceedings 38th CIESM, Congress, 913 April 2007, Istanbul

GOTWAY, C. A. FERGUSON, R. B. HERGERT, G. W. \& PETERSON, T. A. 1996. Comparison of kriging and inversedistance methods for mapping soil parameters. Am. J. Soil Sci. 60: 1237-1247.

GREEN, S. R. KIRKHAM, M. B. CLOTHIER, B. E. 2006. Root uptake and transpiration: from measurements and models to sustainable irrigation. Agrie. Water Manage 86: 165-176.

KIM, Y. EVANS, R.G. \& IVERSEN, W. M. 2008. Remote sensing and control of an irrigation system using a distributed wireless sensor network. IEEE Trans. Instrum. Meas. 57 (7): 13791387.

KRAVCHENKO, A.N., BULLOCK, D.G., 1999. A comparative 
study of interpolation methods for mapping soil properties. J. Agronomy 91, 393-400.

LASLETT, G.M., MCBRATNEY, A.B., PAHL, P.J., Hutchinson, M.F., 1987. Comparison of several spatial prediction methods for soil pH. J. Soil Sci. 38, 325-341.

LEE, W. S. BURKS, T.F. \& SCHUELLER, J. K. 2002. Silage yield monitoring system. Paper number 021165, ASAE Annual Meeting 2002.

MORÁIS, R. BOAVENTURA, I. CORDEIRO, M. \& SALGADO, P. 1996. Ser "odio, P., Couto, C, Solar data acquisition wireless network for agricultural applications. En: Proceedings of the $19^{\text {a1 }}$ Convention of Electrical and Electronics Engineers, Israel, November 5-6, pp. 527-530.

PETERS, R. T. \& EVETT, S. R. 2008. Automation of a centre pivot using the temperature time-threshold method of irrigation scheduling. J. Irrig. Drainage Eng.-ASCE 134(3): 286-291.

SADLER, E. J. EVANS, R. G. STONE, K. C. \& CAMP, C.R. 2005. Opportunities for conservation with precisión irrigation. J. Soil Water Conserv 60: 371-379.

SEELAN, S. K. LAGUETTE, S. CASADY, G. M. SEIELSTAD, G. A. 2003. Remote sensing applications for precision agriculture: a learning community approach. Remote Sens. Environ. 88 : 157169.

VELLIDIS, G. TUCKER, M. PERRY, C. KVIEN, C. BEDNARZ, C. 2008. A real-time wireless smart sensor array for scheduling irrigation. Comput. Electron. Agric. 61: 44- 50.

WEBER, D. ENGLUND, E. 1992. Evaluation and comparison of spatial interpolators. Math. Geol. 24: 381-391. 